\title{
Stimulus Dependence of Neuronal Correlation in Primary Visual Cortex of the Macaque
}

\author{
Adam Kohn and Matthew A. Smith \\ Center for Neural Science, New York University, New York, New York 10003
}

Nearby cortical neurons often have correlated trial-to-trial response variability, and a significant fraction of their spikes occur synchronously. These two forms of correlation are both believed to arise from common synaptic input, but the origin of this input is unclear. We investigated the source of correlated responsivity by recording from pairs of single neurons in primary visual cortex of anesthetized macaque monkeys and comparing correlated variability and synchrony for spontaneous activity and activity evoked by stimuli of different orientations and contrasts. These two stimulus manipulations would be expected to have different effects on the cortical pool providing input to the recorded pair: changing stimulus orientation should recruit different populations of cells, whereas changing stimulus contrast affects primarily the relative strength of sensory drive and ongoing cortical activity. Consistent with this predicted difference, we found that correlation was affected by these stimulus manipulations in different ways. Synchrony was significantly stronger for orientations that drove both neurons well than for those that did not, but correlation on longer time scales was orientation independent. Reducing stimulus contrast resulted in a decrease in the temporal precision of synchronous firing and an enhancement of correlated response variability on longer time scales. Our results thus suggest that correlated responsivity arises from mechanisms operating at two distinct timescales: one that is orientation tuned and that determines the strength of temporally precise synchrony, and a second that is contrast sensitive, of low temporal frequency, and present in ongoing cortical activity.

Key words: noise correlation; visual cortex; orientation; contrast; spontaneous activity; cross-correlogram; signal correlation

\section{Introduction}

When a stimulus is shown repeatedly, trial-to-trial fluctuations in response strength are correlated between neighboring cortical neurons. This correlation, typically characterized by the spike count correlation $r_{\mathrm{sc}}$, interferes with pooling as a strategy to overcome response variability in a population rate code (Zohary et al., 1994; Shadlen and Newsome, 1998). The timing of action potentials between nearby neurons is also often correlated or synchronized, as shown by a peak in the spike train cross-correlogram (CCG). Temporal coding theories propose that synchronous spikes bind the distributed representation of objects (Gray, 1999; Shadlen and Movshon, 1999). Although synchrony and $r_{\text {sc }}$ traditionally have been linked to differing views of how information is encoded by neuronal populations, there is a growing appreciation that they are functionally and mathematically related (Bair et al., 2001; Mazurek et al., 2002).

Both $r_{\mathrm{sc}}$ and synchrony arise from shared input, the source of which is unclear. In the visual system, $r_{\mathrm{sc}}$ is either assumed

Received July 23, 2004; revised Feb. 24, 2005; accepted Feb. 26, 2005.

This work was supported by a Howard Hughes Medical Institute Investigatorship to J. Anthony Movshon and by a grant to Movshon from the National Institutes of Health (EY2017). Additional support was provided by a grant to Peter Lennie (EY04440) and a Core Grant for Vision Research to New York University (EY13079). We thank Wyeth Bair for helpful discussions and, together with Movshon, Simon Schultz, and Brian Lau, for comments on this manuscript.

Correspondence should be addressed to Adam Kohn, Center for Neural Science, New York University, 4 Washington Place, Room 809, New York, NY 10003. E-mail: adamk@cns.nyu.edu.

M. A. Smith's present address: Center for the Neural Basis of Cognition, Carnegie Mellon University, 115 Mellon Institute, 4400 Fifth Avenue, Pittsburgh, PA 15213.

DOI:10.1523/JNEUROSCI.5106-04.2005

Copyright $\odot 2005$ Society for Neuroscience $\quad 0270-6474 / 05 / 253661-13 \$ 15.00 / 0$
(Gawne and Richmond, 1993; Gawne et al., 1996; Reich et al., 2001) or known to be stimulus independent (Zohary et al., 1994; Bair et al., 2001), suggesting that it arises from variations in the state of the cortical network unrelated to the input provided. Synchrony, on the other hand, often is reported to depend on whether a single stimulus is presented or its constituent parts (Gray, 1999). Indirect measures of synchrony also have been shown to depend on basic stimulus parameters such as orientation and speed (Gray and Viana Di Prisco, 1997; Friedman-Hill et al., 2000; Frien et al., 2000). It is unclear, however, how the synchrony between single neurons in primary visual cortex (V1) is affected by such basic stimulus manipulations. Because synchrony and $r_{\mathrm{sc}}$ are related, it is also unclear how the former can be stimulus dependent while the latter is not.

To resolve this discrepancy and to determine how each form of correlation arises, we have measured both $r_{\mathrm{sc}}$ and synchrony between single V1 neurons during spontaneous activity and for activity evoked by stimuli of different orientations and contrasts. Changing stimulus orientation will likely alter the pool of cortical neurons that provides input to the recorded pair, with the predicted effect on correlation depending on the architecture of V1: if the dominant input to V1 cells is feedforward, correlation should be orientation independent; if recurrent connections predominate, correlation should be sensitive to orientation (Ben-Yishai et al., 1995; Series et al., 2004). Changing stimulus contrast, on the other hand, primarily alters the magnitude of the response of the pool, thus allowing us to compare the relative influence of sensory drive and ongoing cortical activity on correlation. 
Characterizing correlation and its stimulus dependence is also important for understanding the computations that can be performed by a neuronal population. For instance, understanding the stimulus dependence of synchrony is necessary for evaluating its role in binding and for determining how it affects the maximal stimulus frequency that can be encoded in a population firing rate (Mazurek and Shadlen, 2002). In addition, theoretical studies suggest that capacity of a population rate code to encode information depends on the magnitude of correlated variability, its relationship to the tuning similarity of two neurons, and its dependence on stimulus drive (Abbott and Dayan, 1999; Sompolinsky et al., 2001; Shamir and Sompolinsky, 2004). Finally, measuring correlation provides a way to test current models for reading out noisy population codes (Pouget et al., 1998), because these make testable predictions about the stimulus dependence of correlation.

We find that stimulus orientation and contrast affect $r_{\text {sc }}$ and CCGs in distinct ways. High-contrast stimuli result in sharper synchronization; low-contrast stimuli have stronger overall correlation but on a broad time scale. This suggests that orientation controls the amount of shared stimulus drive that nearby cells receive, whereas contrast may control internal cortical dynamics.

\section{Materials and Methods}

Recordings were made in 10 cynomolgus (Macaca fascicularis), one bonnet (Macaca radiata), and one pig-tailed (Macaca nemestrina) adult male monkeys. All experimental procedures were approved by the New York University Animal Welfare Committee.

The procedures used in our laboratory for single-unit recording in anesthetized, paralyzed macaque monkeys have been described previously in detail (Cavanaugh et al., 2002). Briefly, animals were premedicated with atropine $(0.05 \mathrm{mg} / \mathrm{kg})$ and diazepam $(1.5 \mathrm{mg} / \mathrm{kg})$ and anesthetized initially with ketamine $\mathrm{HCl}(10 \mathrm{mg} / \mathrm{kg})$. Anesthesia during recording was maintained by intravenous infusion of the opiate anesthetic sufentanil citrate (Sufenta; $4-8 \mu \mathrm{g} \cdot \mathrm{kg}^{-1} \cdot \mathrm{h}^{-1}$ ). To minimize eye movements, the animal was paralyzed with intravenous infusion of vecuronium bromide (Norcuron; $0.1 \mathrm{mg} \cdot \mathrm{kg}^{-1} \cdot \mathrm{h}^{-1}$ ). Vital signs (EEG, electrocardiogram, end-tidal $\mathrm{P}_{\mathrm{CO}_{2}}$, temperature, and lung pressure) were monitored continuously. The pupils were dilated with topical atropine and the corneas protected with gas-permeable contact lenses. Refraction was provided by supplementary lenses.

Recordings were made with an array of seven concentrically arranged platinum/tungsten microelectrodes (Thomas Recording, Giessen, Germany). We made a 10 -mm-diameter craniotomy, centered $10 \mathrm{~mm}$ lateral to the midline and $5-10 \mathrm{~mm}$ posterior to the lunate sulcus, and made vertical electrode penetrations or penetrations normal to the cortical surface. Signals from the microelectrode were amplified, bandpass filtered $(300 \mathrm{~Hz}-10 \mathrm{kHz})$, and fed into a hardware discriminator (BAK Electronics, Germantown, MD) and audio monitor. Spike times were saved with a temporal resolution of $0.25 \mathrm{~ms}$. Most pairs of cells $(90.5 \%)$ were recorded on separate electrodes; pairs were recorded on one electrode only when the waveforms of the cells were clearly distinct.

To allow histological confirmation of the recording site, we made electrolytic lesions at the end of each recording track by passing DC current (typically $2 \mu \mathrm{A}$ for $5 \mathrm{~s}$ ) through the recording electrodes. At the end of the experiment, the monkeys were killed with an overdose of sodium pentobarbital and perfused through the heart with $0.1 \mathrm{M}$ PBS followed by $4 \%$ paraformaldehyde in $0.1 \mathrm{M}$ PBS. Sections $(40 \mu \mathrm{m})$ were stained for Nissl substance with cresyl violet. The close spacing of the electrodes limited our ability to assign lesions to particular electrode tracks. Instead, we used the scatter is lesion location normal to the tracks, together with depth readings from the microdrive system, to calculate the approximate distance between recording sites. Most cells were separated by $300-500 \mu \mathrm{m}$ (maximum tangential separation of $600 \mu \mathrm{m}$ and radial separation of $400 \mu \mathrm{m})$.

Visual stimuli. Stimuli were luminance modulated, drifting sine-wave gratings presented at a frame rate of $100 \mathrm{~Hz}$ using an eight-bit Silicon
Graphics (Mountain View, CA) board operating at a resolution of $1024 \times 731$ pixels. The monitor (Eizo T550) subtended $\sim 10^{\circ}$ of visual angle and had a mean luminance of $\sim 33 \mathrm{~cd} / \mathrm{m}^{2}$. For most cells, we measured, in order, the direction, spatial and temporal frequency, and size tuning for drifting sine-wave gratings (133 of 147 pairs; optimal stimulus parameters in the remaining pairs were determined by hand). After characterizing the stimulus preference of each cell independently, we measured correlation for spontaneous activity and for responses evoked by stimuli of different orientations and contrasts. The spatial and temporal frequency of these test stimuli were set between the preferred values of the two cells or at the value of the cell that responded less vigorously. Stimuli were presented between the receptive field (RF) centers of the cells and covered both RFs. All measurements were made using stimuli presented in a circular aperture to the dominant eye of the less responsive cell. Stimuli were surrounded by a gray field of average luminance.

In the orientation experiments, we presented full-contrast gratings at five orientations that spanned a range between driving the two cells strongly and evoking a weak response; the range spanned $25-122.5^{\circ}$, depending on the tuning bandwidth of the neurons. In the contrast experiments, we used the orientation that was most effective at driving the pair and presented it at four contrasts, typically $1.56,6.25,25$, and $100 \%$, with contrast defined as the difference between the maximum and minimum luminance divided by the sum. For cells with low contrast sensitivity, we adjusted the range of contrasts to extend from 12.5 to $100 \%$ in octave steps. Stimuli were presented for $2.56 \mathrm{~s}$ and separated by $3 \mathrm{~s}$ intervals during which we presented a gray, isoluminant screen. Each stimulus was presented 30-200 times (mean, $80 \pm 3$ trials). Contrast and orientation data were collected in separate blocks of trials. In most experiments, the presentation order of each orientation or contrast was block randomized, but in some experiments $(\sim 10 \%$ of the data), we presented each orientation or contrast repeatedly before testing the next stimulus. We observed no difference between the data from the randomized and nonrandomized stimulus presentation so the data were pooled.

Spike count and timing correlation. We characterized correlation using two metrics, the spike count correlation $r_{\mathrm{sc}}$ and the spike train crosscorrelogram (Perkel et al., 1967), calculated using the methods of Bair et al. (2001).

The $r_{\mathrm{sc}}$, the Pearson correlation coefficient of the evoked spike counts of two cells to the repeated presentation of a particular stimulus, was calculated as follows:

$$
r_{\mathrm{sc}}=\frac{E\left[N_{1} N_{2}\right]-E N_{1} E N_{2}}{\sigma_{N_{1}} \sigma_{N_{2}}},
$$

where $E$ is the expected value, $\sigma$ is the SD of the responses, and $N_{1}$ and $N_{2}$ are the spike counts of cell 1 and 2, respectively. To avoid contamination of our estimates of $r_{\mathrm{sc}}$ by outlier responses, we removed trials on which the response of either neuron was $>3$ SDs different from its mean response (Zohary et al., 1994). Statistical evaluation was performed after converting $r_{\text {sc }}$ to $Z$-scores using the Fisher transformation (Anderson, 1984 ) as follows:

$$
z=\frac{1}{2} \ln \left(\frac{1+r_{\mathrm{sc}}}{1-r_{\mathrm{sc}}}\right) .
$$

To compute the spike train CCG, we represented the spike train of each cell as a binary time series with $1 \mathrm{~ms}$ resolution such that:

$$
x_{j}^{i}(t)= \begin{cases}1, & \begin{array}{l}
\text { if on trial } i, \text { neuron } j \text { fired } \\
\text { an action potential during } \\
\text { the } t \text { th millisecond; } \\
\text { otherwise. }
\end{array}\end{cases}
$$

The CCG was then computed as follows:

$$
C C G(\tau)=\frac{\frac{1}{M} \sum_{i=1}^{M} \sum_{t=1}^{N} x_{1}^{i}(t) x_{2}^{i}(t+\tau)}{\Theta(\tau) \sqrt{\lambda_{1} \lambda_{2}}},
$$


where $M$ is the number of trials, $N$ is the number of bins in the trial, $x_{1}^{i}$ and $x_{2}^{i}$ are the spike trains of neurons 1 and 2 on trial $i, \tau$ is the time lag, and $\lambda_{1}$ and $\lambda_{2}$ are the mean firing rates of the two cells. $\Theta(\tau)$ is the following triangular function:

$$
\Theta(\tau)=T-|\tau|
$$

where $T$ is the trial duration in seconds. This function corrects for the degree of overlap of the two spike trains for each time lag (i.e., that there are $T$ opportunities for simultaneous events in a trial of length $T$ but only $T-1$ opportunities for coincidences at time lags of $1 \mathrm{~ms}$, etc.).

We chose to normalize the CCG by the geometric mean spike rate, as opposed to the product of spike rates or some other factor, for two reasons. First, it is the most commonly used normalization, so it facilitates comparison with previous studies (Mastronarde, 1983; Eggermont, 1994; Eggermont and Smith, 1996; Bair et al., 2001) [similar to the normalization of the joint peristimulus time histogram (Aertsen et al., 1989)]. Second, this normalization provides results that are most comparable with measures of spike count correlation. We explore the reason for this similarity, and how our conclusions about synchrony depend on the normalization we use, in the appendix [available at www.jneurosci.org as supplemental material (references therein: Tolhurst et al., 1983; Vogels et al., 1989)].

All CCGs were corrected for correlation induced by the stimulus by subtracting a shift predictor calculated from trials 1 to $n-1$, with an offset of one trial. Specifically, the shift predictor was calculated as in Equation 4, but with the numerator as the following:

$$
\operatorname{SHIFT}(\tau)=\frac{1}{M-1} \sum_{i=1}^{M-1} \sum_{t=1}^{N} x_{1}^{i}(t) x_{2}^{i+1}(t+\tau) .
$$

We chose to use a shift predictor rather than an all-way shuffle correction, because the latter is affected more strongly by slow fluctuations in neuronal responsivity. In cells in which the firing rate displayed strong temporal modulation (i.e., simple cells), shuffle correction occasionally resulted in artifactual peaks in the CCG caused by bleed through of this modulation. These peaks were clearly distinct from those we report here: they had widths related to the stimulus modulation frequency (several hundred milliseconds wide), and additional peaks were evident at a range of time lags. Shift correction effectively reduced the presence of these relatively rare artifactual peaks but otherwise resulted in CCGs that were essentially identical to those obtained with shuffle correction.

We smoothed the shift-corrected CCGs with a 5 ms kernel ([0.05 0.25 $0.400 .250 .05]$ ) before characterizing CCG shape. We computed CCG peak heights and widths for peaks that exceeded 5 SDs of the noise level at time lags from -1000 to $-950 \mathrm{~ms}$ and from 950 to $1000 \mathrm{~ms}$. Our results were not sensitive to the value of this criterion; we chose a conservative value of $Z=5$ so that only CCGs with clearly visible peaks would be considered. In addition, we present data both above and below this significance criterion. Peak height was defined as the difference between the maximal bin size within $10 \mathrm{~ms}$ of zero time lag and the mean CCG value at large time lags ( $\tau= \pm 950-1000 \mathrm{~ms}$ ), which was usually close to zero. Peak widths were calculated as the difference between the first positive and negative time lag at which the CCG peak reached less than half maximum height (full width at half maximum). For pairs recorded from one electrode, we ignored the -1 to +1 ms bins when calculating CCG peak widths because of our inability to detect simultaneous or nearsimultaneous spiking.

We analyzed every neuronal pair for which we recorded at least 30 repetitions of each stimulus (five pairs for the orientation series and one pair for the contrast series failed this criterion), because our estimates of $r_{\text {sc }}$ would otherwise be unreliable, and the number of spikes recorded would be insufficient for calculating CCGs. We also removed five pairs in the orientation data set and three pairs in the contrast series, because one of the cells in each pair had an abnormally unreliable response, as indicated by a variance-to-mean response ratio $>20$.
To calculate the time scale of correlation, we used the following metric introduced by Bair et al. (2001):

$$
r_{\mathrm{CCG}}(t)=\frac{\sum_{\tau=-t}^{t} \operatorname{CCG}(\tau)}{\sqrt{\left(\sum_{\tau=-t}^{t} A C G_{1}(\tau)\right)\left(\sum_{\tau=-t}^{t} A C G_{2}(\tau)\right)}},
$$

where $t$ is the window of integration. In this case, the CCG in the numerator was calculated as the raw CCG (the numerator of Eq. 4) and was corrected by the all-way shuffle predictor. Autocorrelogram $1\left(\mathrm{ACG}_{1}\right)$ and $\mathrm{ACG}_{2}$, the autocorrelograms of the two neurons, were calculated similarly, but the spike train on each trial was compared with itself. That is, the raw ACG for cell 1 was calculated as follows:

$$
A C G_{1}(\tau)=\frac{1}{M} \sum_{i=1}^{M} \sum_{t=1}^{N} x_{1}^{i}(t) x_{1}^{i}(t+\tau),
$$

and this was corrected by an all-way shuffle predictor. $\mathrm{ACG}_{2}$ was calculated similarly.

Signal correlation analysis. Our measurements of the tuning of the cells for stimulus orientation, spatial and temporal frequency, and size allowed us to explore the relationship between the similarity in receptive field properties of two cells and the magnitude of $r_{\mathrm{sc}}$ or CCG peak height. We quantified this relationship in two ways. First, we calculated the tuning similarity as the signal correlation $r_{\text {signal }}$, which is the Pearson correlation (Eq. 1) between the mean response of each cell to each stimulus. The relationship between $r_{\text {signal }}$ and $r_{\text {sc }}$ or CCG peak height was then characterized by the correlation between these values across all cell pairs. In the second method, we characterized the similarity in tuning as the difference in the preferred orientation or direction, the preferred spatial and temporal frequency, and the degree of RF overlap. These differences were then used as independent variables in a multivariate regression model to predict $r_{\text {sc }}$ or CCG peak height.

The preferred direction for each cell was defined by the vector sum of the responses to 16 test directions $\left(22.5^{\circ}\right.$ steps $)$ as follows:

$$
\text { Preferred direction }=\arctan \left(\frac{\sum_{i=1}^{N} R_{i} \sin \left(\theta_{i}\right)}{\sum_{i=1}^{N} R_{i} \cos \left(\theta_{i}\right)}\right) \text {, }
$$

where $\theta_{i}$ and $R_{i}$ are the direction of motion and response strength, respectively, for stimuli $i=1-16$. We characterized selectivity as the following:

$$
\text { Direction selectivity }=\frac{\left|\sum_{n=1}^{N} R_{n} e^{\left(i \theta_{n}\right)}\right|}{\sum_{n=1}^{N} R_{n}},
$$

which is the length of the summed response vector, normalized by the summed magnitude of all response vectors. The selectivity index varies between 0 , for a cell that responds equally to all directions, and 1 , for a cell that responds to a single direction of motion. Preferred orientation and orientation selectivity were calculated in a similar manner, except that $\theta_{n}$ was replaced by $2 \theta_{n}$ in the equations above and the resultant angle halved. We used the preferred direction for cells for which the direction selectivity was greater than the orientation selectivity and the preferred orientation otherwise.

The preferred temporal and spatial frequency were calculated by fitting a difference-of-Gaussians function to the data, using the STEPIT 
algorithm (Chandler 1969) to minimize the combined $\chi^{2}$ error between the model predictions and the data. From the fitted equation, we defined the optimal stimulus parameter as that which would evoke the strongest predicted response. The difference between the preferred spatial and temporal frequencies of the two cells was then defined in octaves as follows:

$$
\left|\log _{2}\left(\frac{\text { Preferred frequency of cell } 1}{\text { Preferred frequency of cell } 2}\right)\right| .
$$

To measure the receptive field overlap of the two cells, we determined the receptive field center by hand, using a small $\left(\sim 0.3^{\circ}\right)$ patch of optimal grating. We then measured responses to gratings of increasing size and fit the data with a difference-of-error function, using the STEPIT algorithm. The receptive field size of each cell was defined as the maximum of the function in the range tested, and the overlap was defined as the percentage of the smaller RF that was included in the larger RF. The mean receptive field size provided by this approach is approximately twice that provided by hand maps using small bars of light (Cavanaugh et al., 2002). As a result, our estimate of RF overlap is substantially higher than that which would result from mapping with small stimuli.

In our regression analysis, we only used data from pairs for which the fits for both cells accounted for at least $50 \%$ of the variance (123 of 133 pairs for the spatial and temporal frequency data; 114 of 133 pairs for the area data). The variance accounted for by the fits in these cells was on average $90-92 \%$ for each parameter.

\section{Results}

We recorded from 147 pairs of single units in 12 anesthetized, paralyzed macaque monkeys. Because our primary objective was to measure the stimulus dependence of neuronal correlation, we recorded from nearby neurons (typically $<500 \mu \mathrm{m}$ apart) that had similar receptive field properties, because distant or dissimilar neurons tend to fire independently (Nelson et al., 1992; Lee et al., 1998; DeAngelis et al., 1999; Nowak et al., 1999; Bair et al., 2001) and have weak correlation in response variability (Zohary et al., 1994, Lee et al., 1998; Bair et al., 2001; Averbeck and Lee, 2003). We recorded in all cortical layers but biased our population toward complex neurons (76\% of the population) (Skottun et al., 1991). The receptive field properties of the neurons comprising each pair were similar, with a mean difference of $37^{\circ}$ in orientation preference, 0.37 octaves in spatial frequency preference, 0.36 octaves in temporal frequency preference, and a mean receptive field overlap of $75 \%$. The ocular dominance of the two cells was also similar, with a mean difference of 0.83 on the sevenpoint scale of Hubel and Wiesel (1962).

\section{Orientation dependence of spike count correlation}

We evaluated the orientation dependence of $r_{\mathrm{sc}}$, the correlation of evoked spike counts (Eq. 1 in Materials and Methods), by measuring responses to $2.56 \mathrm{~s}$ presentations of full-contrast gratings of five orientations. Figure $1 A$ shows the orientation tuning and range of orientations (thick line) used to measure correlation for an example pair. We chose orientations that spanned a range from driving the pair strongly [geometric mean response of 33 impulses per second (ips)] to evoking a relatively weak response (8.6 ips). Scatter plots of the response of the two cells to multiple presentations of each stimulus are shown in Figure $1 B-E$ as $Z$-scores relative to the mean response for each stimulus. The value of $r_{\text {sc }}$ (text in scatter plots) varied among stimulus condi- tions but did not depend in an obvious way on stimulus orientation or the evoked firing rate. For instance, the correlation for the stimulus that drove both cells strongly (0.24) (Fig. 1D) was similar to that for a stimulus that drove one cell but not the other (0.30) (Fig. 1F).

The data presented in Figure 2 show frequency histograms for $r_{\mathrm{sc}}$ in our population of pairs $(n=100)$, arranged for each pair from the orientation that was most effective at driving the two cells to that which was least effective. We found little relationship between the efficacy of the stimulus and the magnitude of spike count correlation (ANOVA; $p=0.45$ ). Stimuli that drove the pair most strongly ( $42 \pm 2$ ips) had an average correlation of $0.18 \pm 0.03$ (Fig. $2 A$ ), similar to the average $r_{\text {sc }}$ value of $0.19 \pm$ 0.02 for stimuli that evoked the weakest response (12 \pm 1 ips) (Fig. $2 E$ ). The mean $r_{\mathrm{sc}}$ collapsing across all conditions and pairs was 0.20 , a value consistent with previous measurements in the visual system, including those in V1 [0.22 in Gawne et al. (1996) and $\sim 0.25$ in Reich et al. (2001)], middle temporal visual area (MT) [0.19 in Zohary et al. (1994) and 0.20 in Bair et al. (2001)], and inferior temporal cortex [0.23 in Gawne and Richmond (1993)]. Because strong trends between stimulus efficacy and $r_{\text {sc }}$ in individual pairs may go undetected in a population analysis, we also calculated the relationship between the evoked firing rate and $r_{\mathrm{sc}}$ for each pair individually. We found a significant correlation $(p<0.05)$ in only 7 of 100 pairs, three of which were positively correlated and four of which were negatively correlated.

We conclude that there is little relationship between the efficacy of an oriented stimulus and the correlation in trial-to-trial variability of evoked spike count, suggesting that this variability arises from orientation-independent variations in trial-to-trial cortical excitability.

\section{Orientation dependence of spike timing correlation}

Whereas the orientation independence of $r_{\text {sc }}$ agrees well with previous studies (Zohary et al., 1994; Bair et al., 2001), a related form of correlation, spike timing synchrony, has been shown to depend on stimulus drive. However, most studies investigating synchrony between single V1 cortical neurons have focused either on the effect of altering the "gestalt" characteristics of the stimulus (Livingstone, 1996) or have used indirect measurements such as the synchrony of multiunit activity (MUA) (Lamme and Spekreijse, 1998) or the strength of oscillations in single-unit activity, MUA, or the local field potential (LFP) (Gray et al., 1989; Gray and Viana Di Prisco, 1997; Friedman-Hill et al., 2000; Frien et al., 2000). The relationship between the synchronous firing of single neurons and these measurements is unclear. For MUA 


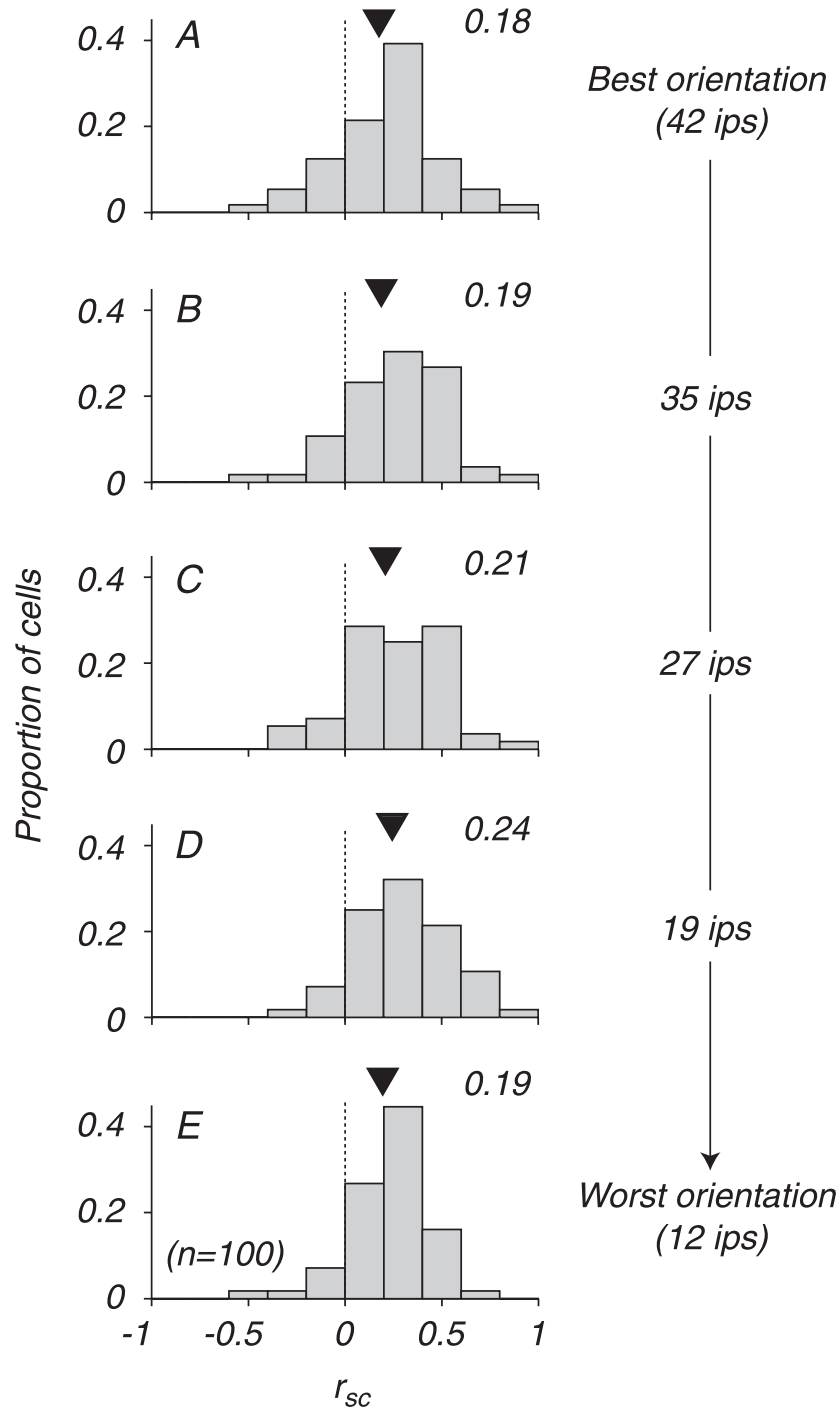

Figure 2. Relationship between firing rate and $r_{s c}$ for stimuli of different orientations. $\boldsymbol{A}-\boldsymbol{E}$, Population histograms of $r_{s,}$, arranged for each cell from the stimulus that drove the pair most effectively to that which evoked the weakest response (average firing rate for each condition indicated at right). The number next to each plot is the mean of the distribution (shown by arrowhead). The distributions are not significantly different.

synchrony, this is because different stimuli might recruit different subpopulations of neurons in the recorded signal. For measures of oscillatory firing, this is because the stimulus dependence of oscillations is controversial (Ghose and Freeman, 1992; Young et al., 1992) and because some studies have failed to find a strong relationship between oscillatory firing and synchronous firing of single neurons (Samonds and Bonds, 2004) (but see Maldonado et al., 2000). To compare the sensitivity of spike count and spike timing correlation between single V1 neurons, we therefore investigated the orientation dependence of synchrony in our population of pairs.

Figure 3, $A$ and $B$, shows CCGs for two pairs of neurons (left and center column), arranged in each case from the most effective to least effective orientation. The CCGs for both pairs had a clear peak centered on zero time lag (inset in top row of traces), as did all but two of the pairs we recorded, indicating that the spike timing correlation was attributable to common synaptic input rather than a direct connection between cells (Moore et al., 1970).
For both pairs, the CCG was clearly dependent on stimulus orientation: the peak of the CCG was smaller for stimuli that evoked a weaker mean firing rate and disappeared entirely for orientations that evoked little response. Figure $3 C$ shows the average CCG for the population of V1 pairs with significant peaks for at least one stimulus condition ( $n=56$ of 100 pairs; see Materials and Methods for criterion), calculated after normalizing data from each pair by the CCG peak height of the most effective orientation. The peak height of the average CCG was smaller for stimuli that evoked weaker responses, but CCG shape was similar across stimulus conditions. Note that for each pair and stimulus condition, the CCG was normalized by the geometric mean firing rate, so that the reduced peak height is not a trivial consequence of the reduced firing rate. We investigate the effect of firing rate on CCG peak height further below, when we consider the effect of stimulus contrast. Other forms of normalization for spike rate are considered in the appendix [available at www.jneurosci.org as supplemental material (references therein: Tolhurst et al., 1983; Vogels et al., 1989)].

We quantified the orientation dependence of spike timing correlation by measuring CCG peak height (maximum value within $10 \mathrm{~ms}$ of $0 \mathrm{~ms}$ time lag) and full width at half height (in milliseconds). The scatter plot in Figure $4 A$ shows the change in CCG peak height for all pairs, comparing the stimulus that evoked the highest firing rate with that which evoked the weakest response. Almost all of the points lie below the identity line, indicating that the peak height was consistently higher for the best-compromise stimulus; the mean peak height decreased from 0.015 to 0.007 coincidences per spike (coinc/spk) (57\%). CCG peak width, on the other hand, was essentially unaffected by changes in stimulus orientation. To ensure meaningful measurements, we computed CCG peak widths only for those CCGs with a $Z$-score of 3 or higher (see Materials and Methods; 55 of 56 pairs had significant CCG peaks for at least two stimulus conditions). The mean peak width was $9.0 \mathrm{~ms}$ for the best-compromise stimulus and $9.7 \mathrm{~ms}$ for stimuli evoking the lowest mean rate $(p=$ 0.63) (Fig. 4B).

We conclude that spike timing correlation is sensitive to stimulus orientation: for orientations ineffective at driving the pair, the CCG peak height is substantially smaller but of similar shape to that for activity evoked by the orientation that evokes the strongest mean response in the pair.

\section{Time scale of correlation}

The dependence of the CCG on stimulus orientation stands in stark contrast to the orientation independence of spike count correlation. This is surprising because both are believed to arise from common synaptic input (Shadlen and Newsome, 1998). Stronger still, the CCG and $r_{\mathrm{sc}}$ are mathematically related (Eq. 7 in Materials and Methods): $r_{\mathrm{sc}}$ is equal to the integral of the CCG, divided by the geometric mean area of the respective autocorrelograms (Bair et al., 2001). Integrating the CCG and ACGs for time lags equal to the trial duration provides spike count correlation values identical to $r_{\text {sc }}$ calculated directly from the total number of spikes evoked during the trial (Figs. 1, 2). Integrating over shorter windows can reveal the time scale over which spike count correlation arises and can clarify why these two forms of correlation have a different sensitivity to stimulus orientation. Importantly, the time scale over which V1 responses are integrated in natural viewing conditions is likely to be on the order of 10-100 ms, because stimulus drive changes frequently (e.g., because of frequent eye movements) and because the neural com- 
putations underlying perception occur quickly (within 150-300 ms) (Thorpe et al., 1996; Johnson and Olshausen, 2003). For pairs with significant spike timing correlation, the orientation dependence of CCG peak height would suggest that $r_{\mathrm{sc}}$ on this more relevant time scale would be largest for the stimulus that evokes the highest mean rate in the pair.

We evaluated the time scale of correlation by integrating the CCG and ACGs at a time resolution of $1 \mathrm{~ms}$, and refer to $r_{\mathrm{sc}}$ calculated in this manner as $r_{\mathrm{CCG}}$. We averaged the $r_{\text {CCG }}$ curves for each stimulus condition across our population of pairs and scaled the final value to one to facilitate comparison of the time course. The value of $r_{\mathrm{CCG}}$, as a function of the size of the integration window, for the most effective stimulus is shown as a dotted line in Figure $5 A$. The value of $r_{\mathrm{CCG}}$ increased with the size of the integration window and reached its final value for windows of 50-100 ms, a value approximately consistent with previous measurements in MT (32 ms) (Bair et al., 2001) but shorter than the values reported for cat V1 (500-1000 ms) (Reich et al., 2001). For less effective orientations (dashed line for the stimulus that evoked the next highest firing rate, followed by solid lines of increasing thickness), the respective $r_{\mathrm{CCG}}$ curves had lower values for small integration windows (e.g., $<10 \mathrm{~ms}$ ), reflecting the loss of area in the central peak of the CCG. We calculated the time at which each average $r_{\mathrm{CCG}}$ curve reached $90 \%$ of its final value, indicated by the dots on the curves in Figure $5 \mathrm{~A}$. As the stimulus orientation became more effective at driving the pair, the $90 \%$ rise time decreased from $231 \mathrm{~ms}$ for the least effective stimulus to $53 \mathrm{~ms}$ for the most effective stimulus.

A consequence of the loss in CCG peak height for stimuli evoking weak mean firing in the pair is that $r_{\mathrm{CCG}}$ for brief response epochs will be orientation dependent. To illustrate this, we replotted the relationship between $r_{\mathrm{CCG}}$ and the time scale of integration for the most (gray trace) and the least (black trace) effective stimulus in Figure $5 B$. These curves are not normalized; therefore, $r_{\mathrm{CCG}}$ can be read directly off the ordinate. For an integration window of $1 \mathrm{~ms}, r_{\mathrm{CCG}}$ for the best-compromise stimulus was approximately three times larger than for the least effective stimulus. The difference between the $r_{\text {CCG }}$ curves is maintained for integration windows less than $\sim 200 \mathrm{~ms}$; over this range, the $r_{\mathrm{CCG}}$ curve for the least effective stimulus resembles the $r_{\mathrm{CCG}}$ curve for the bestcompromise stimulus but with a negative offset as a result of the loss of area on short time scales. Consistent with the orientation independence of $r_{\mathrm{sc}}$, the $r_{\mathrm{CCG}}$ curves converge for integration windows of $>200 \mathrm{~ms}$, and the value for the largest integration window (2600 ms) was not significantly different for the two conditions. These data suggest that orientationsensitive common input determines the strength of correlation on time scales relevant for cortical computation but that an orientation-independent mechanism dominates on the time scale of hundreds of milliseconds to seconds.
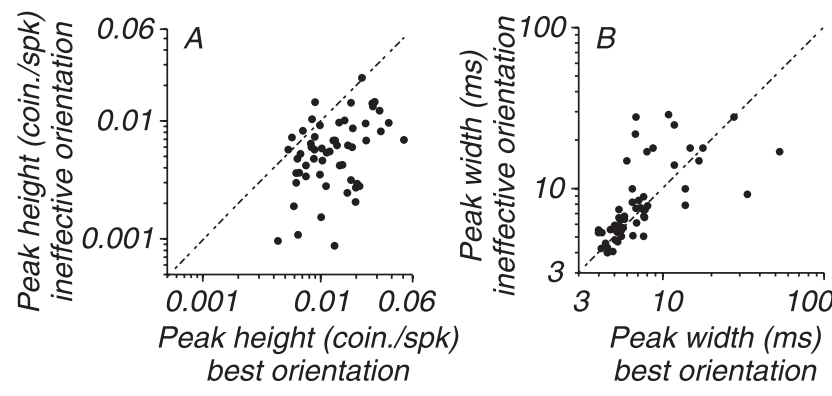

Figure 4. CCG peak height and width for the most effective and least effective orientations. $A$, Pair-by-pair comparison of CCG peak height for stimuli evoking the highest firing rate with those evoking the lowest rate. Most of the points are below the identity line indicating a decrease in CCG amplitude for stimuli that evoke a weak response. $\boldsymbol{B}$, Pair-by-pair comparison of CCG peak width, defined as the width at half-maximal amplitude. Peak width is essentially insensitive to changes in stimulus orientation. coin./spk, Coincidences per spike.

\section{Magnitude and time scale of correlation for unsynchronized pairs}

The analysis in the preceding sections focused on the stimulus dependence of spike timing correlation and its effect on $r_{\mathrm{CCG}}$ in pairs with significant CCG peaks (56 of 100 pairs). In the remaining pairs, synchronization was weak or absent for all stimulus conditions, as illustrated by the average CCGs for the most effective ( $33 \pm 3$ ips; gray trace) and least effective ( $9 \pm 1$ ips; black trace) stimulus conditions shown in Figure $6 \mathrm{~A}$. Because of the relationship between $r_{\mathrm{sc}}$ and the CCG, pairs without significant spike timing correlation should have weak $r_{\mathrm{CCG}}$ values at all time scales, and $r_{\mathrm{CCG}}$ should be stimulus independent. The $r_{\mathrm{CCG}}$ plots shown in Figure $6 B$ show that this was indeed the case. First, there was no difference between the $r_{\mathrm{CCG}}$ curves for effective (thick gray 


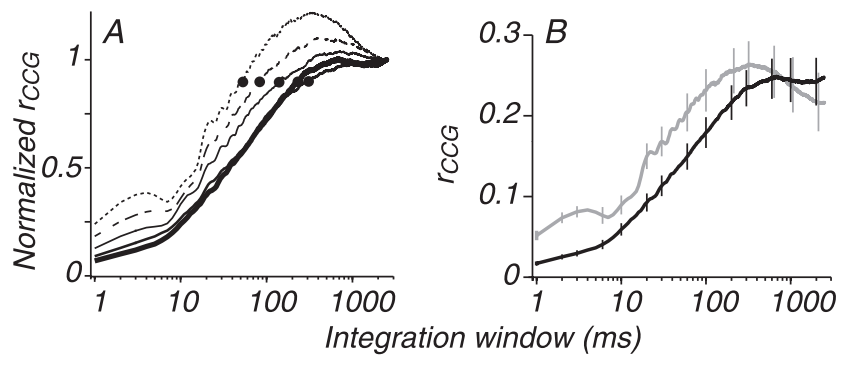

Figure 5. Effect of stimulus orientation on the time scale of correlation. A, Normalized $r_{\mathrm{CCG}}$ plots calculated by integrating the $C(G$ and ACGs for a range of integration windows. Each curve is the average $r_{\text {CCG }}$ (across 56 pairs) for a given stimulus condition; the dotted line is for the most effective orientation, and the dashed line is for the next highest firing rate, followed by lines of increasing thickness. Dots indicate the time at which $r_{\text {CGG }}$ for each condition reaches $90 \%$ of its final value. $\boldsymbol{B}$, Nonnormalized average $r_{c c G}$ curves for the most effective (gray) and least effective (black) stimuli. The value of $r_{c c G}$ is different on short time scales, but the curves converge for integration windows $>300 \mathrm{~ms}$. Error bars indicate SEM.
$A$

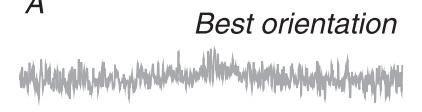

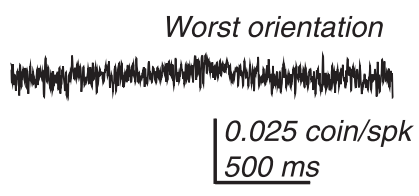

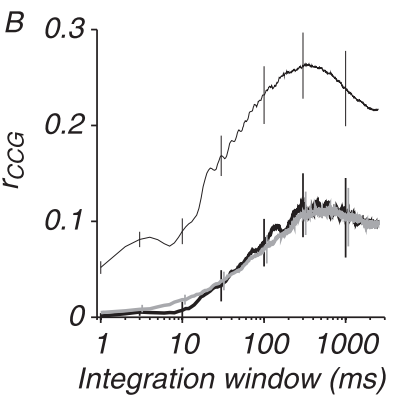

Figure 6. Correlation in pairs without significant spike timing correlation. A, Average CCGs for most effective (gray) and least effective (black) stimuli in 44 pairs of V1 neurons for which we observed no significant peak in the $C\left(G\right.$. $B$, The average $r_{\text {CCG }}$ curves for these pairs reveals no difference between the most effective (gray) and least effective (black) orientations. The ${ }_{{ }_{C c G}}$ for the most effective orientation in pairs with significant $C(G$ peaks, identical to that in Figure $5 B$, is replotted as a thin, black line for comparison. Correlation is weaker on all time scales in pairs without CCG peaks. coin/spk, Coincidences per spike. Error bars indicate SEM.

line) and ineffective (thick black line) orientations for pairs without CCG peaks, and the correlation for both conditions was nearly zero at short time scales $\left(r_{\mathrm{CCG}}<0.03\right.$ for integration windows $<30 \mathrm{~ms}$ ). Second, the correlation for the best-compromise orientation in pairs with significant CCG peaks (thin black line; replotted from Fig. 5B) was substantially larger than for pairs without CCG peaks (thick gray and black traces) at all time scales. For the largest integration window, the $r_{\mathrm{CCG}}$ values for pairs with significant CCG peaks was $0.22 \pm 0.03$ compared with $0.11 \pm$ 0.02 for pairs without $(p=0.007)$.

In summary, pairs with significant CCG peaks, presumably those that share substantial common input, display a significant orientation dependence of spike timing correlation. As a result, the response variability of neurons is significantly more correlated for effective than ineffective orientations for response epochs of $<100 \mathrm{~ms}$. At long time scales, there is little stimulus dependence evident. In pairs without spike timing synchronization, presumably those that share less common input, the correlation of trial-to-trial response variability is significantly lower, and no stimulus dependence is evident. In a later section, we will show that the strength of synchronization and $r_{\mathrm{sc}}$ are related to the similarity in receptive field properties of the two neurons.

\section{Effect of stimulus contrast on correlation}

Whereas synchrony and correlated response variability on short time scales are stronger for orientations that evoke a robust re-

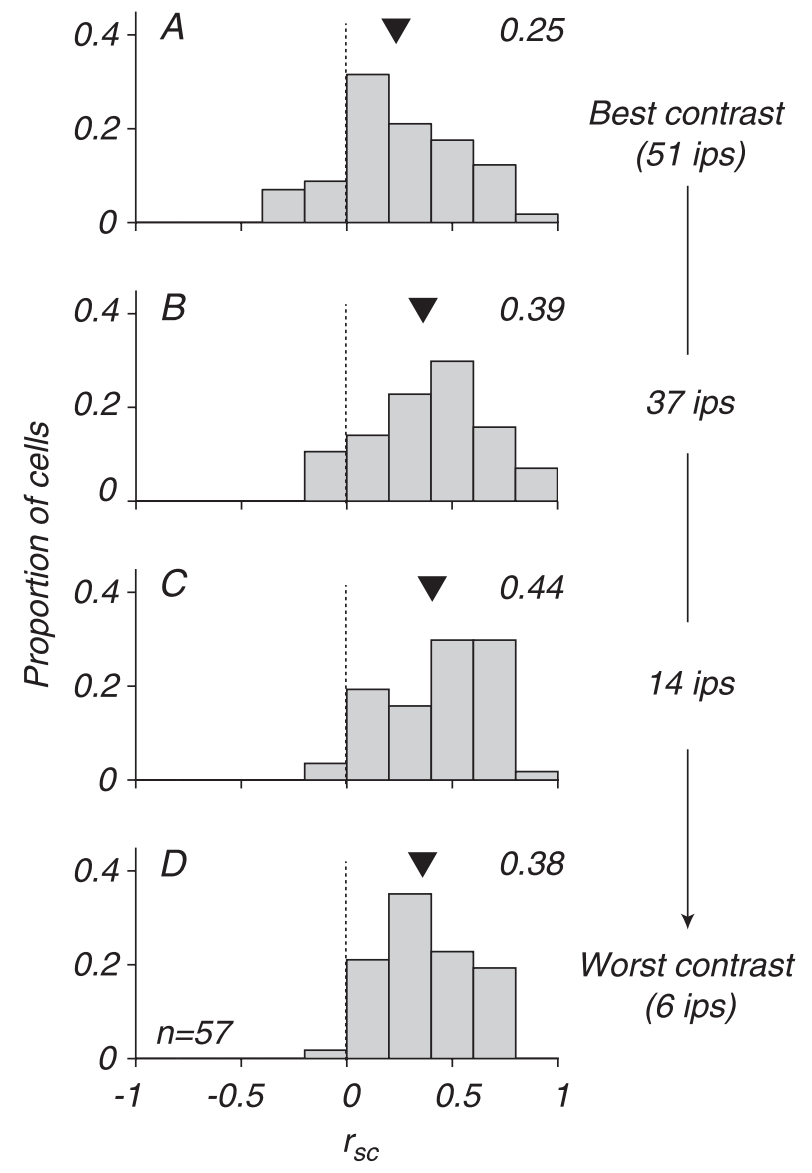

Figure 7. Relationship between firing rate and $r_{\mathrm{sc}}$ for stimuli of different contrasts. $\boldsymbol{A}-\boldsymbol{D}$, Population histograms of $r_{\text {sc }}$ arranged for each cell from the stimulus that drove the pair most effectively to that which evoked the weakest response, which was almost always the highest to lowest contrast. The number next to each plot is the mean of the distribution (shown by arrowhead). The average $r_{\text {sc }}$ increases significantly as stimulus contrast is lowered.

sponse in both neurons, correlation is primarily orientation independent on longer time scales and thus determined by some other source. To determine whether correlation on long time scales is at all stimulus sensitive or simply reflects ongoing cortical activity, we measured the effect of reducing stimulus contrast on correlation, a manipulation that would be expected to alter the strength of the evoked cortical response rather than to alter strongly which neurons are recruited by the stimulus. Comparing the effect of stimulus orientation and contrast on correlation is also important for technical reasons: the measured strength of synchrony is notoriously sensitive to firing rate, even when the strength of common input is fixed (Melssen and Epping, 1987; Binder and Powers, 2001; Turker and Powers, 2002; Shu et al., 2003). This makes it difficult to know whether effects on synchrony reflect orientation-dependent changes in the amount of common input or simply a change in our ability to detect this synchrony because of the rate dependence of the CCG. By comparing the effects of orientation and contrast on synchrony, we can distinguish between these possibilities.

We begin by computing $r_{\mathrm{sc}}$ for the best-compromise grating (i.e., the orientation that evokes the highest mean firing rate in the pair) presented at a range of contrasts, typically 1.25 to $100 \%$ in double-octave steps. The dependence of $r_{\mathrm{sc}}$ calculated from the entire response epoch on stimulus contrast for our population of V1 pairs ( $n=57$ pairs) is shown in Figure 7, with the data arranged from the strongest to the weakest stimulus (almost always 
the highest to lowest contrast). The mean $r_{\mathrm{sc}}$ value was significantly larger for intermediate- and low-contrast stimuli than high-contrast stimuli (ANOVA; $p<0.001$ ). The dependence of $r_{\mathrm{sc}}$ on contrast was not linear; rather, $r_{\mathrm{sc}}$ was similarly high over a wide range of low to moderate contrasts but dropped strongly at maximum contrast. That the correlation in trial-to-trial response variability was weaker for full-contrast stimuli suggests that strong stimulus drive can reduce the correlation caused by ongoing cortical activity (for similar findings in area MT, see Cardoso de Oliveira et al., 1997; Bair et al., 2001).

The elevated $r_{\mathrm{sc}}$ values at low contrast suggest that the area under the CCG increases as stimulus contrast is lowered, as opposed to the sharp loss in peak height observed as the orientation of a highcontrast stimulus is changed. The two example pairs in Figure 8, $A$ and $B$ (left and center column), indicate that this was indeed the case. At low stimulus contrast (bottom rows), CCG peak height was reduced in amplitude, but there was a compensatory increase in peak width. The population average CCG $(n=57)$ (Fig. $8 C$ ) reveals both a substantial increase in peak width at lower contrasts and a loss in the amplitude of the narrow central peak. On a pair-by-pair basis, there was a significant drop in CCG peak amplitude: the mean amplitude decreased by $46 \%$ as stimulus efficacy decreased. Distinct from the effect of altering stimulus orientation, however, there was a substantial increase in the CCG peak width for low-contrast stimuli, with the mean width at half maximum more than doubling from 11.5 to $24.0 \mathrm{~ms}(p=0.006)$.

In principle, the increase in $r_{\mathrm{sc}}$ and the appearance of a broad CCG at low contrast could arise because of adaptation. For instance, the response of both cells to a low-contrast stimulus could be suppressed by a preceding high-contrast stimulus as a result of rapid changes in contrast gain (Bonds, 1991). The adaptationinduced fluctuations in response strength would inflate $r_{\mathrm{sc}}$ and could lead to artifactual CCG peaks (Brody, 1999). To control for the potential effects of adaptation, we compared the correlation for a low-contrast stimulus presented in isolation with that measured when the same stimulus was interleaved with high-contrast stimuli ( $n=12$ pairs). The $r_{\text {sc }}$ values were nearly identical for the two cases, with a mean value of 0.52 and 0.53 for isolated and interleaved stimuli, respectively, and the CCGs were also similar (width of 41 vs $33 \mathrm{~ms}$; height of 0.016 vs 0.015 coinc/spk). In an additional seven pairs of cells, we measured responses to noninterleaved high- and low-contrast stimuli and found that consistently larger $r_{\mathrm{sc}}$ values $(0.28$ for high contrast vs 0.47 for low contrast) and broader CCGs (9.2 vs $24.8 \mathrm{~ms}$ ) for the low-contrast stimuli. Finally, the correlation we observe at low contrast agrees well with that of spontaneous activity, as discussed below. Together, these results suggest strongly that adaptation does not cause the elevated correlation at low contrast.

The increase in CCG peak width at low contrast suggests a significant increase in the time scale of correlation for these stimuli. Figure $9 A$ shows the population-normalized $r_{\mathrm{CCG}}$ curves for high-contrast stimuli (dotted line) and for stimuli of decreasing

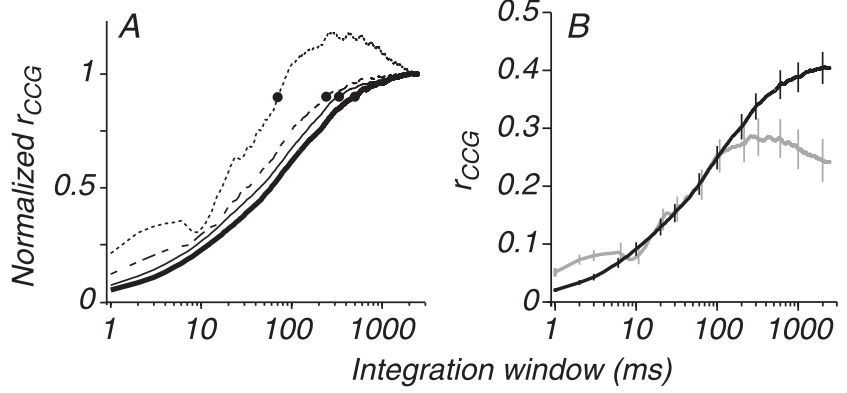

Figure 9. Effect of stimulus contrast on the time scale of correlation. $\boldsymbol{A}$, Normalized $r_{\text {ccG }}$ plots (across 57 V1 pairs) for each stimulus contrast; the dotted line is for the highest contrast, followed by the dashed line and solid lines of increasing thickness. The curve for the highest contrast has the most area on a short time scale and reaches it maximal value most quickly. Less effective stimuli have more area at long time scales and reach their final value more slowly. Dots indicate the $90 \%$ rise time of $r_{\text {CCG. }}$ B, Nonnormalized $r_{C C G}$ curves for high- (gray) and lowcontrast (black) stimuli. The value of $r_{\mathrm{CCG}}$ is larger for high-contrast stimuli because these stimuli evoke a large, narrow CCG peak, but the additional CCG area at long time scales for low-contrast stimuli results in larger $r_{C \mathrm{CG}}$ values for these stimuli on long time scales. Error bars indicate SEM.

contrast (dashed line and lines of increasing thickness). The time scale of correlation decreased monotonically as the stimuli became more effective: the time at which $r_{\text {CCG }}$ reached $90 \%$ of its final value decreased from $499 \mathrm{~ms}$ (thick solid line) for the lowest contrast to $70 \mathrm{~ms}$ (dotted line) for full contrast. In Figure $9 B$, unnormalized $r_{\mathrm{CCG}}$ plots for high- and low-contrast stimuli allow direct comparison of values on different time scales. The correlation on very brief time scales $(<10 \mathrm{~ms})$ was higher for fullcontrast stimuli (gray trace) because of the relatively large sharp peak in the CCG for this condition. For intermediate time scales (10-100 ms), the increased CCG peak width at low contrast compensated for the loss of area in the narrow central peak, and the $r_{\text {CCG }}$ values are essentially identical. Finally, for integration windows greater than $\sim 100 \mathrm{~ms}, r_{\mathrm{CCG}}$ was significantly larger for the 

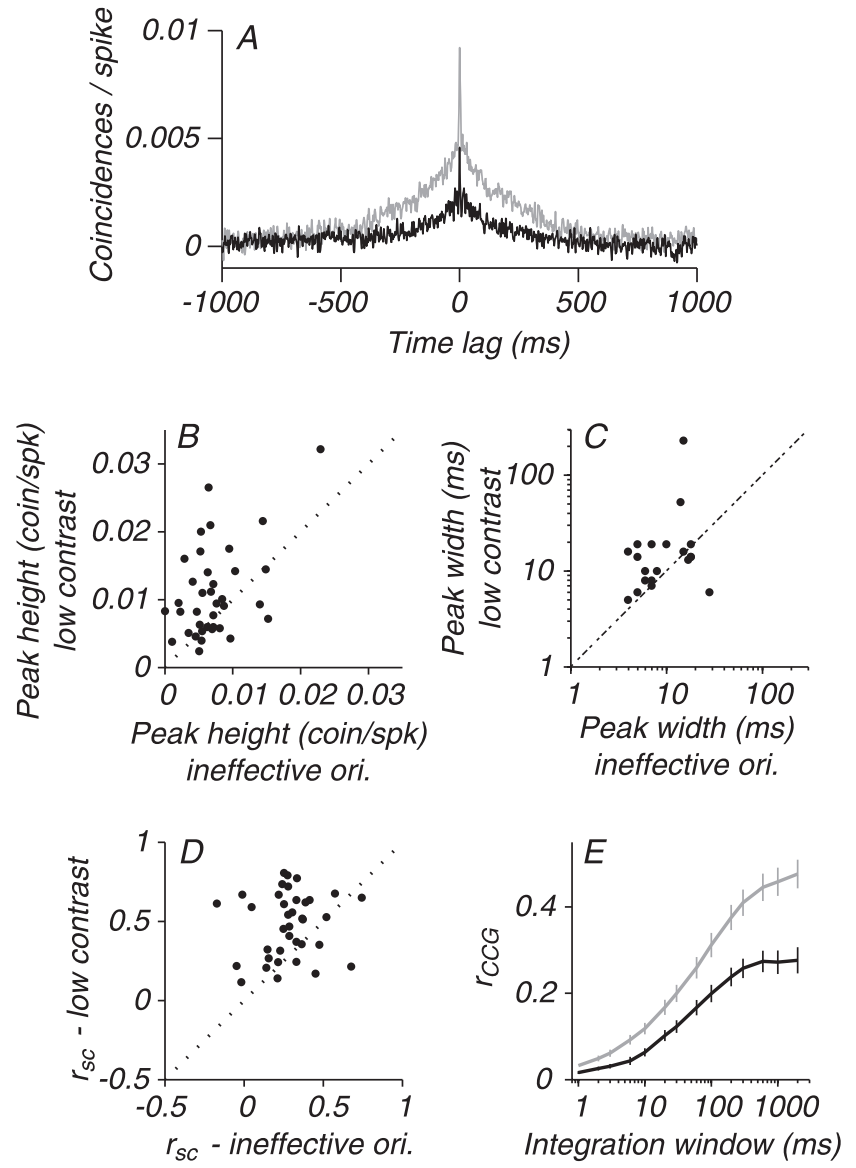

Figure 10. Direct comparison of correlation for low-contrast and ineffectively oriented stimuli that evoke similar firing rates. $\boldsymbol{A}$, Average CCGs for low-contrast (gray) and ineffectively oriented (black) stimuli. CCGs are larger and broader at low contrast. $\boldsymbol{B}$, Pair-by-pair comparison of CCG peak height for low-contrast and ineffective orientations (ori.). Low-contrast CCGs have larger peaks. C, Pair-by-pair comparison of CCG peak width. Low-contrast CCGs are broader. D, Comparison of the spike count correlation. The $r_{\text {sc }}$ values are consistently larger for low-contrast stimuli. $\boldsymbol{E}$, Comparison of the time scale of correlation for low-contrast (gray) and ineffective orientations (black). The average $r_{\mathrm{CCG}}$ for low-contrast stimuli is larger on all time scales. coin/ spk, Coincidences per spike. Error bars indicate SEM.

low-contrast data, consistent with the results shown in Figure 7. Thus, although the $90 \%$ rise times increase for both low-contrast and ineffective orientations, they do so for different reasons. For changes in stimulus orientation, the effect is attributable primarily to a subtractive shift in the $r_{\mathrm{CCG}}$ curves caused by reduced sharp synchrony. For contrast, the change in rise time is caused by an increase in the value of $r_{\mathrm{CCG}}$ for large integration windows.

Clearly, stimulus contrast and orientation affect correlation differently. However, the preceding analysis involved overlapping but not identical sets of neuronal pairs, and, more importantly, the range of firing rates explored in the contrast experiments was larger than that for orientation (primarily because many V1 neurons respond vigorously to nonpreferred orientations) (Ringach et al., 2002). To make a more direct comparison, we evaluated the stimulus dependence of correlation for 37 pairs of neurons for which we obtained both types of data and for which the CCG peak was significant. We compared the correlation observed at the least effective orientation (mean response of 15 ips) with that of the contrast that evoked the most similar firing rate (mean response of $16 \mathrm{ips}$ ). The average CCG for the low-contrast stimulus (Fig. $10 \mathrm{~A}$, gray trace) was larger and broader than the average CCG evoked by a high-contrast stimu- lus, the orientation of which evoked a similar firing rate (black trace). The scatter plots in Figure 10, $B$ and $C$, show the difference in CCG peak height and width, respectively, on a pair-by-pair basis for the two conditions. Peak height was significantly larger ( 0.011 vs 0.007 coinc/spk; $p=0.003$ ), and the width at half maximum was substantially broader ( 27.3 vs $10.1 ; p=0.024)$ for the low-contrast stimulus. The better maintained CCG peak height and increased peak width resulted in significantly higher $r_{\mathrm{sc}}$ values for the low-contrast stimulus ( 0.48 vs $0.28 ; p<0.001$ ) (Fig. $10 D$ ), a difference that was apparent at all time scales (Fig. $10 \mathrm{E}$, gray $r_{\mathrm{CCG}}$ curve for low contrast vs black for ineffective orientation).

In summary, our results suggest that correlated response variability and spike timing correlation are affected differently by changes in stimulus orientation and contrast. Stimulus orientation controls the source of input that causes sharp synchrony, but correlation on longer time scales is orientation independent. This latter source of correlation is, however, affected by stimulus drive: high-contrast stimuli suppress it.

\section{Correlation of spontaneous activity}

The strong correlation we observe for activity evoked by lowcontrast stimuli suggests that cortical neurons receive substantial common input in the absence of effective stimulus drive. This proposal is consistent with recent optical imaging studies of the spatial distribution of spontaneous activity in V1 (Arieli et al., 1996; Tsodyks et al., 1999), which found that population activity preceding spontaneous spikes was similar to that evoked by an optimal stimulus. Thus, it appears that spontaneous spikes occur because the neurons that provide input to the recorded cell are active, a description that applies equally well to evoked activity. More recently, Fizer et al. (2004) reported that the spatial and temporal structure of ongoing cortical activity was relatively insensitive to stimulus drive. To provide a direct comparison of the correlation of evoked and spontaneous activity, we recorded spontaneous activity for periods of 15-30 min in neuronal pairs that had an appreciable spontaneous firing rate (mean rate of 7.2 ips; $n=42$ pairs).

Consistent with the imaging study of Tsodyks et al. (1999), we typically found a significant peak in both the spontaneous and stimulus-evoked CCG (48\% of pairs) or in neither (26\%). In 10\% of pairs, we observed a significant peak in the spontaneous CCG but not the evoked data, and in $17 \%$ we observed the opposite, although in many of these cases, the CCGs just failed to meet our significance criterion. Figure $11, A$ and $B$, illustrates for two pairs of V1 neurons the differences between CCGs calculated from spontaneous activity (gray traces at bottom) with those for activity evoked by full-contrast gratings, the orientation of which evoked either strong (top traces) or weak (middle traces) mean responses. First, the peak height of CCGs for activity evoked by the best-compromise stimulus was usually either the same as (left column) or larger (right column) than for spontaneous activity. Second, the peak height of CCGs for activity evoked by ineffectively oriented stimuli could be either smaller (left column) or similar (right column) in amplitude to those for spontaneous activity. Finally, the width of spontaneous CCGs was consistently broader than for either high-contrast stimulus.

As suggested by the broad width and substantial amplitude of the spontaneous CCGs, the mean $r_{\mathrm{sc}}$ for spontaneous activity in all pairs was relatively strong $(0.29 \pm 0.04$; calculated from $2.56 \mathrm{~s}$ epochs). When we limited our analysis to pairs for which the spontaneous CCGs were significant $(n=24)$, the mean $r_{\text {sc }}$ was 0.38 , similar to the value at low contrast for pairs with significant CCG peaks and almost twice as high as the correlation of activity 
$A$
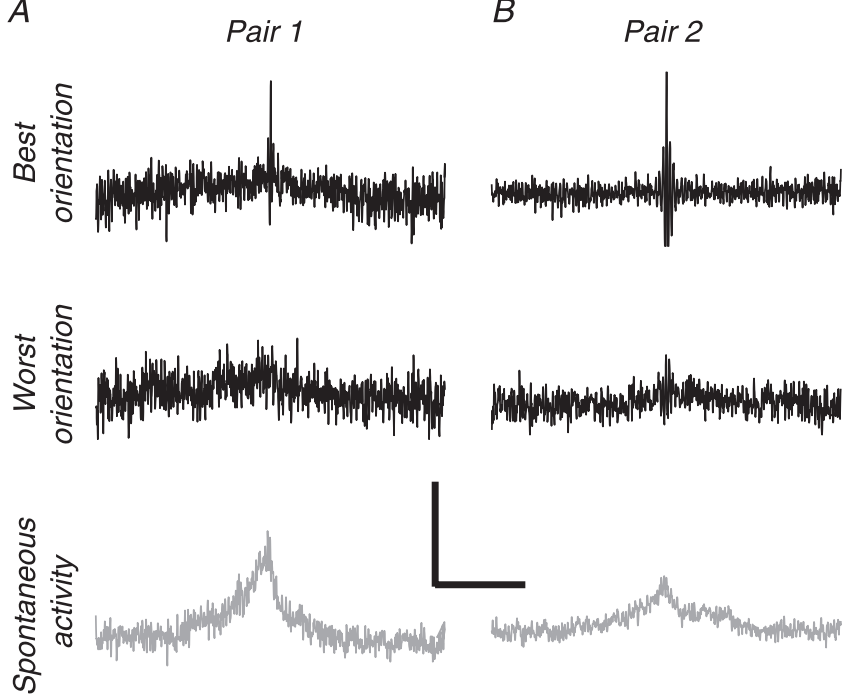

C
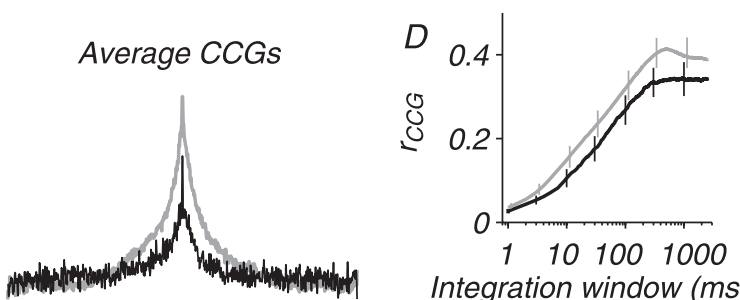

Integration window (ms)

Figure 11. Comparison of spontaneous and evoked correlation. A, CCGs for activity evoked by the most effective orientation (top), the least effective orientation (middle), and spontaneous activity (bottom; gray) for an example V1 pair. The spontaneous CCG is larger and broader than that for the best-compromise stimulus. The least effectively oriented stimulus disrupts the broad CCG observed during spontaneous activity. $\boldsymbol{B}$, Same as in $\boldsymbol{A}$ for a pair for which the $C(G$ of spontaneous activity and activity evoked by the least effective orientation have a similar amplitude; the spontaneous CCG is substantially broader than those for activity evoked by highcontrast stimuli. C, Average CCGs ( $n=24$ pairs) for spontaneous activity (gray) and activity evoked by ineffective orientations (black). Although the firing rate was stronger for the evoked activity, the CCG peak amplitude was smaller. Vertical calibration is 0.01 coincidences per spike (coinc/spk) for $A, 0.02$ coinc/spk for $B$, and 0.01 coinc/spk for $C$; horizontal calibration is $500 \mathrm{~ms}$. $D$, Time scale of correlation for spontaneous (gray) and ineffective (black) stimuli. The $r_{\text {cGG }}$ curves show that correlation is stronger for the spontaneous activity at all time scales. Error bars indicate SEM.

evoked by high-contrast stimuli. The mean peak height in this subset of cells was 0.012 coinc/spk, between the mean height for most effective and least effective orientations $(0.015$ and 0.007 coinc/spk, respectively). Finally, the mean spontaneous CCG peak width was $69 \mathrm{~ms}$, slightly larger than that observed during the presentation of low-contrast stimuli. A direct comparison between the average CCG for the least effective high-contrast stimuli and spontaneous activity recorded in the same pairs is shown in Figure 11C. The greater CCG peak height and width results in a larger $r_{\mathrm{CCG}}$ for spontaneous activity (gray trace) at all time scales (Fig. 11D).

Thus, we find that ongoing cortical activity is strongly correlated and that this correlation is not attributable to unspecific changes in cortical excitability. First, correlation of spontaneous activity arises from loose synchrony of spike timing on the time scale of hundreds of milliseconds. Second, synchronized spontaneous activity typically occurs in pairs of neurons whose evoked activity is also synchronized, consistent with previous reports that the spatial structure of activity in cortex is similar for spontaneous and evoked activity (Arieli et al., 1996; Tsodyks et al., 1999). Finally, the signal that gives rise to this correlation can be

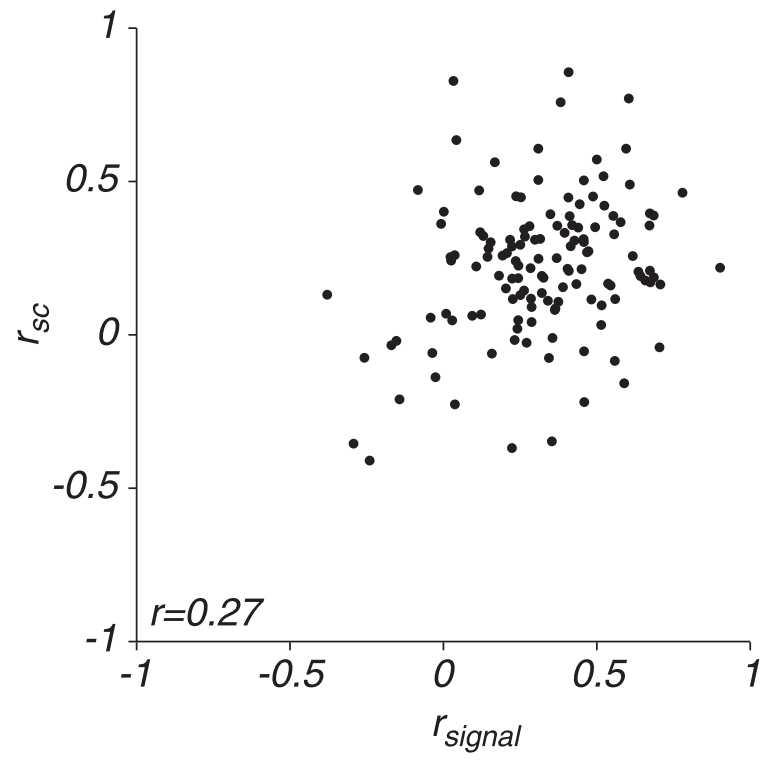

Figure 12. Relationship between signal correlation and spike count correlation. There is a weak relationship ( $r=0.27$ ) between $r_{\text {signal }}$ and $r_{\text {sc }}$, indicating that neurons with similar receptive field properties tend to have stronger correlation in trial-to-trial variability.

suppressed by high-contrast stimuli, even when such stimuli evoke little response in the recorded pair.

\section{Relationship between response correlation and receptive field similarity}

If the correlation documented in the previous sections is related to common input to the pair of neurons, there should be some relationship between receptive field properties and the overall magnitude of correlation. Specifically, because the RF properties of a neuron are determined primarily by its inputs, cells with similar RF properties are likely to receive more common input than those with dissimilar preferences and should thus be more strongly correlated. Our measurements of orientation, spatial and temporal frequency, and size tuning, used to determine the optimal stimulus parameters for each pair, provided an opportunity to investigate how $r_{\mathrm{sc}}$ and spike timing correlation depend on the similarity in tuning between the two cells. A useful measure of tuning similarity is $r_{\text {signal }}$, which is the correlation between the mean responses of two neurons to the same stimulus set. Because it depends completely on the stimulus set with which it is measured, the magnitude of $r_{\text {signal }}$ is by itself ambiguous (i.e., two neurons can be viewed as having similar or dissimilar tuning depending on the richness of the stimulus set presented), but the relationship between $r_{\text {signal }}$ and $r_{\text {sc }}$ remains informative.

We evaluated the relationship between the $r_{\mathrm{sc}}$ and $r_{\text {signal }}$ by calculating the signal correlation for each stimulus parameter and comparing it to $r_{\mathrm{sc}}$ across our population of pairs. We used the average $r_{\mathrm{sc}}$ measured across all orientations or, for pairs for which we only measured responses to stimuli of varying contrasts, at high contrast. We found a positive but weak relationship between $r_{\text {signal }}$ for any particular stimulus parameter and $r_{\mathrm{sc}}$ (orientation: $r=0.10, p=0.21$; spatial frequency: $r=0.13, p=0.12$; temporal frequency: $r=0.17, p=0.05$; receptive field size: $r=0.27, p=$ 0.001 ), but when we characterized the similarity in tuning with a single $r_{\text {signal }}$ value for all stimulus parameters, the correlation between $r_{\text {signal }}$ and $r_{\text {sc }}$ was stronger and statistically significant $(r=0.27, p=0.001)$ (Fig. 12). We also found a significant relationship between $r_{\text {signal }}$ and CCG peak height $(r=0.32$; $p<$ 
Table 1. Relationship between signal and noise correlation: parameters of multivariate regression analysis

\begin{tabular}{llc}
\hline & Value/slope & $p$ value \\
\hline$y$ intercept & 0.37 & $<0.0001$ \\
Orientation & $-0.0015 /$ degree & 0.047 \\
Spatial frequency & $-0.063 /$ octave & 0.35 \\
Temporal frequency & $-0.11 /$ octave & 0.08 \\
RF overlap & $-0.054 /$ percentage overlap & 0.39 \\
\hline
\end{tabular}

$0.001)$. The relationship between $r_{\text {sc }}$ and $r_{\text {signal }}$ in $\mathrm{V} 1(r=0.27)$ is similar to that documented for nearby neurons in motor and parietal cortex $(\sim 0.15-0.5)$ (Lee et al., 1998) and in macaque supplementary motor area (0.35) (Lee, 2003). In MT, Bair et al. (2001) found a stronger correlation of 0.61 , but this may be because the pairs were recorded on a single electrode and thus particularly close together (Lee et al., 1998). Our data, on the other hand, may underestimate the relationship between $r_{\text {signal }}$ and $r_{\mathrm{sc}}$ in $\mathrm{V} 1$, because we recorded few cells with negative signal correlation (Fig. 12, points in the left half) and because we did not explore all stimulus parameters for which V1 neurons are selective (e.g., color and disparity tuning).

In a complementary analysis, we investigated the relationship between tuning similarity and response correlation using a multivariate regression model. The independent parameters in the regression analysis were the differences in preferred orientation, spatial and temporal frequency, and the RF overlap of the two cells (see Materials and Methods). The most informative model included all of the variables and predicted $10 \%$ of the variance in $r_{\mathrm{sc}}(r=0.31 ; p=0.03)$. The coefficients for each variable were negative so that larger differences in preference predicted lower $r_{\mathrm{sc}}$ values, but only the term for orientation preference was significant at the $p=0.05$ level (Table 1). Previous studies have suggested that neurons with similar ocular dominance are more likely to fire synchronously than those with different preferences (Kruger and Aiple, 1988; Hata et al., 1991). For a subset of neurons $(n=55)$, we measured ocular dominance quantitatively and included the difference in the response ratio in the two eyes (in octaves) as an additional variable in the model. This model explained a higher percentage of the variance of $r_{\mathrm{sc}}(29 \%$; $p=0.02)$, and orientation and ocular dominance were the most important factors.

We conclude that there is a relationship between the tuning similarity of two cells and both the correlation in their response variability and the strength of spike timing correlation. A simple explanation for these data are that common input to a pair of neurons gives rise to similar tuning but also correlated response variability and a tendency to fire synchronously. It is the modulation of the strength and temporal characteristics of this common input by stimulus orientation and contrast that presumably gives rise to the stimulus-dependent correlation we observe.

\section{Discussion}

We have shown that stimulus contrast and orientation have distinct effects on the correlation of single V1 neurons. During spontaneous activity and for weak to moderate stimulus drive, trial-to-trial variability of similarly tuned neurons is often strongly correlated and synchronized with loose temporal precision. High-contrast stimuli reduce correlation on long time scales, make synchronous firing more precise, and either enhance or suppress synchrony strength depending on whether the stimulus drives the pair strongly. Together, our results suggest that correlation arises from two distinct mechanisms: a low-frequency component attributable to ongoing activity, which dominates correlated variability on long time scales and which is suppressed by strong stimulus drive; and a temporally precise component, which is orientation tuned and causes sharp synchronization.

\section{Comparison with previous studies}

Previous studies have assumed that spike count correlation in V1 is stimulus independent (Gawne et al., 1996; Reich et al., 2001). We find instead that for response epochs of several seconds, $r_{s c}$ is orientation independent but increases for low-contrast stimuli; on the time scale of tens of milliseconds, $r_{\mathrm{sc}}$ is strongest for orientations that drive both cells strongly but primarily independent of stimulus contrast.

Several studies have investigated the dependence of timing correlation between single neurons in V1 on basic stimulus parameters. Snider et al. (1998) found that monosynaptic connections (CCGs with peaks offset from zero) between neurons in cat V1 were more sensitive to stimulus orientation than contrast. Samonds and Bonds (2004) showed that common-input CCGs are larger for effectively oriented stimuli. Llampl et al. (1999) showed that membrane potential fluctuations between pairs of V1 neurons are correlated and stimulus dependent, also consistent with our data. Our study extends these findings by comparing the sensitivity of common input CCGs to stimulus orientation and contrast, by comparing spontaneous and evoked correlation, and by relating the stimulus dependence of synchrony to changes in the spike count correlation over a range of time scales.

Remaining studies of V1 synchrony have used indirect measures or have explored the role of synchrony in binding a distributed representation. Because we used single gratings, our data does not directly evaluate the role of synchrony in binding, but it does suggest that synchrony can be partly predicted by whether a stimulus provides potent common drive to two neurons. We also found substantial synchrony, albeit on a broad time scale, in the absence of any stimulus drive. Both findings suggest that activity will often be synchronized even when there is no need for binding.

\section{Functional implications}

Previous measurements of $r_{\mathrm{sc}}(\sim 0.2)$ were based on stimuli presented for several seconds and thus overestimate its value on time scales relevant for sensory information processing (10-100 ms). In a simple pooling model, lower $r_{\mathrm{sc}}$ values mean that the population signal-to-noise ratio will improve when a larger number of neurons are averaged (Zohary et al., 1994; Bair et al., 2001). In addition, approximately one-half of our pairs lacked significant synchronization (Fig. 6), so that $r_{\mathrm{CCG}}$ on short time scales was nearly zero. Although the preferences of these neurons was more different on average than for neurons that were strongly synchronized, the tuning of the cells was usually sufficiently similar so that a single grating could drive both cells. Any stimulus should thus excite a neuronal population whose variability is essentially uncorrelated during short epochs; pooling over such cells could significantly reduce response variability.

In the pooling model, the fidelity of the neuronal population, the ability to encode time-varying stimuli in the population firing rate, depends critically on the time scale of correlation. Mazurek and Shadlen (2002) showed that for an $r_{\mathrm{sc}}$ of 0.2 that arises solely from synchronous spikes, the population is unable to follow temporal modulations over $50 \mathrm{~Hz}$, but this cut-off frequency increases if correlation arises on a slower time scale. We find that the correlation for high-contrast stimuli arises over tens of milliseconds, so that the population could encode stimuli that vary at $>200 \mathrm{~Hz}$. At low stimulus contrast, both the time scale and the magnitude of $r_{\mathrm{sc}}$ increase, so the fidelity should remain limited. 
More sophisticated analysis of population coding has shown that correlation can either improve or impair stimulus encoding, relative to the performance of an uncorrelated population of cells (Vogels, 1990; Abbott and Dayan, 1999; Sompolinsky et al., 2001). The accuracy of the code decreases if similarly tuned neurons are correlated more strongly than dissimilar cells, as we find. For some algorithms for reading population codes, however, stimulus-dependent correlation can improve markedly the accuracy of the population code (Shamir and Sompolinsky, 2004). The net effect of the V1 correlation we report will thus depend on the degree to which subsequent stages of cortical processing can read out stimulus dependent correlation.

Our V1 findings allow us to evaluate the physiological plausibility of current models for reading population codes. Pouget et al. (1998) and Deneve et al. (1999) proposed a recurrent neural network to recover the information available in a population of noisy input neurons. The model predicts that the response covariance between neurons should be negative for orientations that fall where the slope of the tuning curves are different and positive for stimuli that fall on a common flank. Our results are inconsistent with this suggestion, because correlation is either orientation independent (over seconds) or maximal for stimuli that drive both cells well (on time scales $<100 \mathrm{~ms}$ ), typically at an orientation between the peak preference of the cells, where the slopes of the tuning curves are different. We checked this directly by calculating the average correlation for stimuli that fell where the product of the slopes was either negative or positive but found no difference between the two ( 0.20 vs $0.22 ; p=0.8)$.

Finally, the stimulus dependence of correlation can be used to constrain models of V1 circuitry. A strictly feedforward model predicts CCGs that are orientation independent (Ben-Yishai et al., 1995), clearly inconsistent with our findings. Models with recurrent cortical connections predict that the tails of the CCG will be negative for stimuli between the peaks of the tuning curves and positive where the slopes of the tuning curves are similar. We did observe a tendency for correlation at long time scales to decrease for the most effective stimuli (Fig. 5), but this effect was weaker than that predicted by Ben-Yishai et al. (1995) and unrelated to the slopes of the tuning curves. Our results are thus not consistent with either implementation of the Ben-Yishai et al. model. More complex models of V1 circuitry, presumably those with realistic patterns of recurrent connectivity and spontaneous cortical activity, may provide a better match.

\section{Mechanisms}

Our data suggest that at least two sources of input contribute to correlated responsivity: one of low frequency that is present during spontaneous activity and is sensitive to stimulus contrast and a second that depends on stimulus orientation. The mechanism responsible for the low-frequency component present in spontaneous activity is not clear. In the visual system, it may be partly caused by slow correlation in the activity of retinal ganglion cells (60-100 ms) (Mastronarde, 1989; Brivanlou et al., 1998), which is reduced by increasing light intensity, just as increasing stimulus contrast reduces CCG peak width in V1. Low frequency correlation may also involve correlated transitions to "UP" (depolarized) states in cortical neurons (Llampl et al., 1999). In any case, the low frequency correlation of spontaneous activity is unlikely to be caused by anesthesia because (1) broad CCGs have been reported in the visual cortex of awake monkeys (Gochin et al., 1991); (2) broad correlation of spontaneous subthreshold activity is related to the simultaneously recorded LFP but not to more global measures such as the EEG (Llampl et al., 1999); and (3) the
LFP is dominated by low frequencies in both anesthetized and awake animals (Amzica and Steriade, 1995; Llampl et al., 1999; Leopold et al., 2003).

As contrast increases, the CCG peak narrows, and its amplitude either increases or decreases depending on stimulus efficacy. At the network level, tighter synchronization may be related to shifts in the LFP power spectra toward high frequencies (Llampl et al., 1999; Lee, 2003; Henrie, 2004). Cellular mechanisms may also contribute. Azouz and Gray (2003) reported that during depolarization, action potentials are preferentially triggered by high-frequency inputs. Stimulus drive also increases membrane conductance (Borg-Graham et al., 1998; Anderson et al., 2000), thus making the cell less sensitive to low-frequency input. Such changes in conductance also occur for ineffective stimuli, which could explain the disruption of spontaneous synchrony by highcontrast stimuli that fail to evoke a strong response (Hirsch et al., 1998; Anderson et al., 2000). Interestingly, $r_{\mathrm{sc}}$ changed primarily between full contrast and the next lowest contrast (typically 0.25 ), reminiscent of the signal that causes response saturation at high contrasts (Carandini et al., 1997). Finally, the magnitude of sharp synchrony depends in a straightforward way on stimulus orientation: gratings that evoke strong responses result in substantial synchrony; orientations that evoke weak responses result in weak synchrony. Although it is unclear how CCG peak height relates to the amount of common input [see Appendix, available at www. jneurosci.org as supplemental material (references therein: Tolhurst et al., 1983; Vogels et al., 1989)], a simple explanation for the orientation dependence of synchrony is that stimuli that drive two neurons strongly provide more common input that those that evoke weak responses.

\section{References}

Abbott LF, Dayan P (1999) The effect of correlated variability on the accuracy of a population code. Neural Comput 11:91-101.

Aertsen AM, Gerstein GL, Habib MK, Palm G (1989) Dynamics of neuronal firing correlation: modulation of "effective connectivity." J Neurophysiol 61:900-917.

Amzica F, Steriade M (1995) Short- and long-range neuronal synchronization of the slow $(<1 \mathrm{~Hz})$ cortical oscillation. J Neurophysiol 73:20-38.

Anderson JS, Carandini M, Ferster D (2000) Orientation tuning of input conductance, excitation, and inhibition in cat primary visual cortex. J Neurophysiol 84:909-926.

Anderson T (1984) An introduction to multivariate statistical analysis. New York: Wiley.

Arieli A, Sterkin A, Grinvald A, Aertsen A (1996) Dynamics of ongoing activity: explanation of the large variability in evoked cortical responses. Science 273:1868-1871.

Averbeck BB, Lee D (2003) Neural noise and movement-related codes in the macaque supplementary area. J Neurosci 23:7630-7641.

Azouz R, Gray CM (2003) Adaptive coincidence detection and dynamic gain control in visual cortical neurons in vivo. Neuron 37:513-523.

Bair W, Zohary E, Newsome WT (2001) Correlated firing in macaque visual area MT: time scales and relationship to behavior. J Neurosci 21:1676-1697.

Ben-Yishai R, Lev Bar-Or R, Sompolinsky H (1995) Theory of orientation tuning in visual cortex. Proc Natl Acad Sci USA 92:3844-3848.

Binder MD, Powers RK (2001) Relationship between simulated common synaptic input and discharge synchrony in cat spinal motoneurons. J Neurophysiol 86:2266-2275.

Bonds AB (1991) Temporal dynamics of contrast gain in single cells of the cat striate cortex. Vis Neurosci 6:239-255.

Borg-Graham LJ, Monier C, Fregnac Y (1998) Visual input evokes transient and strong shunting inhibition in visual cortical neurons. Nature 393:369-373.

Brivanlou IH, Warland DK, Mesiter M (1998) Mechanisms of concerted firing among retinal ganglion cells. Neuron 20:527-539.

Brody CD (1999) Correlations without synchrony. Neural Comput 11:1527-1535.

Carandini M, Heeger DJ, Movshon JA (1997) Linearity and normalization 
in simple cells of the macaque primary visual cortex. J Neurosci 17:8621-8644.

Cardoso de Oliveira S, Thiele A, Hoffman K-P (1997) Synchronization of neuronal activity during stimulus expectation in a direction discrimination task. J Neurosci 17:9248-9260.

Cavanaugh JR, Bair W, Movshon JA (2002) Nature and interaction of signals from the receptive field center and surround in macaque V1. J Neurophysiol 88:2530-2546.

Chandler JD (1969) Subroutine STEPIT: finds local minima of a smooth function of several parameters. Behav Sci 14:81-82.

DeAngelis GC, Ghose GM, Ohzawa I, Freeman RD (1999) Functional micro-organization of primary visual cortex: receptive field analysis of nearby neurons. J Neurosci 19:4046-4064.

Deneve S, Latham PE, Pouget A (1999) Reading population codes: a neural implementation of ideal observers. Nat Neurosci 2:740-745.

Eggermont JJ (1994) Neural interactions in cat primary auditory cortex II. Effects of sound stimulation. J Neurophysiol 71:246-270.

Eggermont JJ, Smith GM (1996) Neural connectivity only accounts for a small part of neural correlation in auditory cortex. Exp Brain Res 110:379-391.

Fizer J, Chiu C, Welliky M (2004) Small modulation of ongoing cortical dynamics by sensory input during natural vision. Nature 431:573-578.

Friedman-Hill S, Maldonado PE, Gray CM (2000) Dynamics of striate cortical activity in the alert macaque: I. Incidence and stimulus dependence of gamma-band neuronal oscillations. Cereb Cortex 10:1105-1116.

Frien A, Eckhorn R, Bauer R, Woelbern T, Gabriel A (2000) Fast oscillations display sharper orientation tuning that slower components of the same recordings in striate cortex of the awake monkey. Eur J Neurosci 12:1453-1465.

Gawne TJ, Richmond BJ (1993) How independent are the messages carried by adjacent inferior temporal cortical neurons? J Neurosci 13:2758-2771.

Gawne TJ, Kjaer TW, Hertz JA, Richmond BJ (1996) Adjacent visual neurons share about $20 \%$ of their visual information. Cereb Cortex 6:482-489.

Ghose GM, Freeman RD (1992) Oscillatory discharge in the visual system: does it have a functional role? J Neurophysiol 68:1558-1574.

Gochin PM, Miller EK, Gross CG, Gerstein GL (1991) Functional interactions among neurons in inferior temporal cortex of the awake macaque. Exp Brain Res 84:505-516.

Gray CM (1999) The temporal correlation hypothesis of visual feature integration: still alive and well. Neuron 24:31-47.

Gray CM, Viana Di Prisco G (1997) Stimulus dependent neuronal oscillations and local synchronization in striate cortex of the alert cat. J Neurosci 17:3239-3253.

Gray CM, Konig P, Engel AK, Singer W (1989) Oscillatory responses in cat visual cortex exhibit inter-columnar synchronization which reflects global stimulus properties. Nature 338:334-337.

Hata Y, Tsumoto T, Tamura H (1991) Horizontal interactions between visual cortical neurons studied by cross-correlation analysis in the cat. J Physiol (Lond) 441:593-614.

Henrie JA (2004) On ensemble activity in primary visual activity. $\mathrm{PhD}$ thesis, Center for Neural Science, New York University.

Hirsch JA, Alonso J-M, Reid RC, Martinez LM (1998) Synaptic integration in striate cortical simple cells. J Neurosci 18:9517-9528.

Hubel DH, Wiesel TN (1962) Receptive fields, binocular interaction and functional architecture in the cat's visual cortex. J Physiol (Lond) 160:106-154.

Johnson JS, Olshausen BA (2003) Timecourse of neural signatures of object recognition. J Vis 3:499-512.

Kruger J, Aiple F (1988) Multimicroelectrode investigation of monkey striate cortex: spike train correlations in the infragranular layers. J Neurophysiol 16:2381-2396.

Lamme VA, Spekreijse H (1998) Neuronal synchrony does not represent texture segregation. Nature 396:362-366.

Lee D (2003) Coherent oscillations in neuronal activity of the supplementary motor area during a visuomotor task. J Neurosci 23:6798-6809.

Lee D, Port NL, Kruse W, Georgopoulos AP (1998) Variability and correlated noise in the discharge of neurons in motor and parietal areas of the primate cortex. J Neurosci 18:1161-1170.

Leopold DA, Murayama Y, Logothetis NK (2003) Very slow activity fluctuations in monkey visual cortex: implications for functional brain imaging. Cereb Cortex 13:422-433.

Livingstone MS (1996) Oscillatory firing and interneuronal correlations in squirrel monkey striate cortex. J Neurophysiol 75:2467-2485.

Llampl I, Reichova I, Ferster D (1999) Synchronous membrane potential fluctuations in neurons of the cat visual cortex. Neuron 22:361-374.
Maldonado PE, Friedman-Hill S, Gray CM (2000) Dynamics of striate cortical activity in the alert macaque: II. Fast time scale synchronization. Cereb Cortex 10:1117-1131.

Mastronarde DN (1983) Correlated firing of cat retinal ganglion cells. I. Spontaneously active inputs to X- and Y-cells. J Neurophysiol 49:303-324.

Mastronarde DN (1989) Correlated firing of retinal ganglion cells. Trends Neurosci 12:75-80.

Mazurek ME, Shadlen MN (2002) Limits to the temporal fidelity of cortical spike rate signals. Nat Neurosci 5:463-471.

Melssen WJ, Epping WJM (1987) Detection and estimation of neural connectivity based on crosscorrelation analysis. Biol Cybern 57:403-414.

Moore GP, Segundo JP, Perkel DH, Levitan H (1970) Statistical signs of synaptic interactions in neurons. Biophys J 10:876-900.

Nelson JI, Salin PA, Munk MH-J, Bullier J (1992) Spatial and temporal coherence in cortico-cortical connections: a cross-correlation study in areas 17 and 18 in the cat. Vis Neurosci 9:21-37.

Nowak LG, Munk MHJ, James AC, Girard JP, Bullier J (1999) Crosscorrelation study of the temporal interactions between areas V1 and V2 of the macaque monkey. J Neurophysiol 81:1057-1074.

Perkel DH, Gerstein GL, Moore GP (1967) Neuronal spike trains and stochastic point processes II. Simultaneous spike trains. Biophys J 7:419-440.

Pouget A, Zhang K, Deneve S, Latham PE (1998) Statistically efficient estimation using population coding. Neural Comput 10:373-401.

Reich DS, Mechler F, Victor JD (2001) Independent and redundant information in nearby cortical neurons. Science 294:2566-2568.

Ringach DL, Shapley RM, Hawken MJ (2002) Orientation selectivity in macaque V1: diversity and laminar dependence. J Neurosci 22:5639-5651.

Samonds JM, Bonds AB (2004) Gamma oscillation maintains stimulus structure-dependent synchronization in cat visual cortex. J Neurophysiol 93:223-236.

Series P, Latham PE, Pouget A (2004) Tuning curve sharpening for orientation selectivity: coding efficiency and the impact of correlations. Nat Neurosci 7:1129-1135.

Shadlen MN, Movshon JA (1999) Synchrony unbound: a critical evaluation of the temporal binding hypothesis. Neuron 24:67-77.

Shadlen MN, Newsome WT (1998) The variable discharge of cortical neurons: implications for connectivity, computation and information coding. J Neurosci 18:3870-3896.

Shamir M, Sompolinsky H (2004) Nonlinear population codes. Neural Comput 16:1105-1136.

Shu Y, Hassentaub A, Badoual M, Bal T, McCormick DA (2003) Barrages of synaptic activity control the gain and sensitivity of cortical neurons. J Neurosci 23:10388-10401.

Skottun BC, DeValois RL, Grosof DH, Movshon JA, Albrecht DG, Bonds AB (1991) Classifying simple and complex cells on the basis of response modulation. Vision Res 31:1079-1086.

Snider RK, Kabara JF, Roig BR, Bonds AB (1998) Burst firing and modulation of functional connectivity in cat striate cortex. J Neurophysiol 80:730-744.

Sompolinsky H, Yoon H, Kang K, Shamir M (2001) Population coding in neuronal systems with correlated noise. Phys Rev E Stat Nonlin Soft Matter Phys 64:051904.

Thorpe S, Fize D, Marlot C (1996) Speed of processing in the human visual system. Nature 381:520-522.

Tolhurst DJ, Movshon JA, Dean AF (1983) The statistical reliability of signals in single neurons in cat and monkey visual cortex. Vision Res 23:7775-7785.

Tsodyks M, Kenet T, Grinvald A, Arieli A (1999) Linking spontaneous activity of single cortical neurons and the underlying functional architecture. Science 286:1943-1946.

Turker KS, Powers RK (2002) The effects of common input characteristics and discharge rate on synchronization in rat hypoglossal motoneurones. J Physiol (Lond) 541:245-260.

Vogels R (1990) Population coding of stimulus orientation by striate cortical cells. Biol Cybern 64:25-31.

Vogels R, Spileers W, Orban GA (1989) The response variability of striate cortical neurons in the awake behaving monkey. Exp Brain Res 77:432-436.

Young MP, Tanaka K, Yamane S (1992) On oscillating neuronal responses in the visual cortex of the monkey. J Neurophysiol 67:1464-1474.

Zohary E, Shadlen MN, Newsome WT (1994) Correlated neuronal discharge rate and its implications for psychophysical performance. Nature 370:140-143. 